\title{
Crystallizing sugar science
}

\author{
Recent ballot initiatives instituting a tax on sugary drinks in the US, alongside related efforts by other countries and \\ support from the World Health Organization, bring to the forefront the need for greater scientific insight into how \\ sugars affect metabolic health.
}

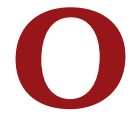
n 8 November, there was a closely contested election to select the next president of the US. But overlooked in the media frenzy over the battle for the presidency were local initiatives in a few cities with a potentially important impact on public health. Namely, residents in parts of California's Bay Area, which includes San Francisco, as well as those in Boulder, Colorado, passed by wide margins a measure to institute a per-ounce tax on sugar-sweetened beverages. These votes followed on the heels of a proclamation in mid-October by the World Health Organization in support of such a tax in countries across the globe (http://go.nature. com/2gcwrwe). Notably, such levies have already been approved in other US cities, such as Berkeley, California, and Philadelphia, Pennsylvania, as well as in other countries, such as France, Mexico, the UK, the Philippines and South Africa. Opponents of this type of tax say that it is unfair to the economically disadvantaged and that it may hurt employment. Proponents, however, argue that it can lead to marked reductions in the consumption of sugary beverages, and thus help in the fight against obesity and diabetes. And the monies raised can contribute to health and education programs that deal with these conditions.

We do not want to get into the public-policy debate here. But in the same way that cigarette taxes were not generally accepted until the science linking smoking and cancer became overwhelming, it is unlikely that a tax on sugar-sweetened beverages will gain wide traction until there is strong science to back such policy decisions. So, what do we know about sugar metabolism, and what do we need still to figure out?

When we talk about sugars for human consumption, we are referring typically to glucose, fructose and sucrose. Fructose is sensed as the sweetest of these and is usually added to food and drinks either in the form of high-fructose corn syrup, which contains a mixture of monomers of glucose and fructose, or as sucrose, which is a disaccharide of glucose and fructose. But these sugars are not metabolized in a similar manner. Whereas glucose is transported into most tissues equivalently-but especially, into the skeletal muscle, liver, adipose tissue and brain-studies in animals and humans suggest that ingested fructose is largely taken up and metabolized in the liver and the intestine.

Upon transport into liver cells, fructose is phosphorylated immediately by fructokinase; thus, as observed in rats and humans, a rapid elevation of fructose can cause a transient, but critical, decrease in intracellular adenosine triphosphate (ATP) and phosphate in liver cells. This depletion subsequently results in the activation of AMP deaminase (AMPD) and an ensuing enzymatic pathway that leads to elevated uric acid levels. AMPD and uric acid both act on the liver to promote fat accumulation in that organ, which contributes to insulin resistance. In addition to these effects on liver metabolism, high fructose levels have been shown to induce leptin resistance in animal studies. And a high uric acid level has been linked to elevated blood pressure in humans and in animals, possibly acting through decreases in renal function or activation of the renin-angiotensin system. Insulin resistance, fatty liver and elevated blood pressure are all hallmarks of metabolic syndrome.

Although the evidence in cell culture and from rodent studies is fairly compelling, what is not clear is how well these findings translate to human metabolism. One study has reported that reducing sugary-beverage intake might have benefits on blood pressure. Small clinical trials have also reported that lowering uric acid levels by inhibiting its synthesis with allopurinol might also have a benefit on blood pressure, including an increase in pressure caused by excess fructose consumption. But larger, better-designed, placebocontrolled trials need to be performed. Some small studies also suggest that lowering uric acid might have benefits on other features of the metabolic syndrome (such as insulin resistance) and/or on kidney disease. Larger trials are now under way to determine the effects of lowering uric acid on cardiovascular and renal complications.

In rodents, it is clear that it is the dose and speed of fructose consumption that matters. If too much is consumed too quickly, then the transient drop in intracellular ATP in hepatocytes occurs, which results in the noncaloric side-chain reaction that generates excess uric acid. So perhaps the proposal by former New York City Mayor Michael Bloomberg to institute a citywide ban on servings of sugary drinks larger than 16 ounces at restaurants, movie theaters, stadiums and arenas was, in hindsight, the way to go. That measure, although adopted by the city's Board of Health in 2012, was ultimately overturned in the courts.

An alternative option to reducing individual serving sizes would be to replace high-fructose corn syrup or sucrose with glucose. However, glucose is not very sweet, and studies in animals suggest that at high levels, it is converted to fructose by the body. Artificial sweeteners could be another option, but although they are considered generally safe, it is unclear what their systemic effects might be if more widely consumed on a population level. Alternatively, people could just consume fewer added sweeteners, which is the goal of the sugary-drinks tax. The only certainty is that we need more science to better understand the likely health effects of our options. 